\title{
Effects of downregulated expression of microRNA-187 in gastric cancer
}

\author{
WENJING CHEN $^{1}$, YONGXIN CUI ${ }^{1,2}$, JIULONG WANG $^{1}$, YUQING YUAN ${ }^{1}$, \\ XIANGWEI SUN $^{1}$, LIANG ZHANG ${ }^{1}$, SHURONG SHEN ${ }^{1}$ and JUN CHENG ${ }^{1}$
}

\author{
${ }^{1}$ Department of Gastrointestinal Surgery, The First Affiliated Hospital of Wenzhou Medical University, Wenzhou, \\ Zhejiang 325035; ${ }^{2}$ Department of General Surgery, Zhoukou Central Hospital, Zhoukou, Henan 466000, P.R. China
}

Received September 28, 2015; Accepted November 4, 2016

DOI: $10.3892 /$ etm.2018.6318

\begin{abstract}
RNAs (miRNAs) are involved in cancer development and progression, and have regulatory roles as tumor suppressors or oncogenes. Although aberrant expression of miR-187 has been observed in several types of cancer, its pathophysiological role and relevance to tumorigenesis in gastric cancer (GC) remains unknown. In the present study, the expression and biological role of miR-187 was investigated in 32 specimens of GC tissues and their adjacent non-tumorigenic controls, and the association between miR-187 expression and clinical features of GC were analyzed further. Kaplan-Meier survival curves determined the clinical significance of miR-187 expression in GC. Following transfection with miR-187 mimics, the biological functions of miR-187 were determined by cell proliferation and cell cycle assays. Moreover, following transfection with miR-187 mimics, the targets regulated by miR-187 were investigated using western blotting. Luciferase reporter assays confirmed whether miR-187 regulated MAD2 mitotic arrest deficient-like 2 (MAD2L2) and stomatin (EPB72)-like 2 (STOML2) expression. The data of the present study revealed that miR-187 was significantly downregulated in GC compared with adjacent non-tumorigenic counterparts. Furthermore, decreased expression of miR-187 correlated with cell differentiation $(\mathrm{P}<0.05)$, TNM staging $(\mathrm{P}<0.05)$ and poor prognosis in GC patients. Functional studies indicated that miR-187 overexpression evidently inhibited MGC-803 cell proliferation in vitro and altered the cell cycle by arresting cells in the $\mathrm{G}_{0} / \mathrm{G}_{1}$ phase. In addition, the luciferase assay and western blotting revealed that MAD2L2 and STOML2 were targeted by miR-187. In conclusion, it is suggested that miR-187 functions as a tumor suppressor in GC, and is important in the
\end{abstract}

Correspondence to: Dr Jun Cheng, Department of Gastrointestinal Surgery, The First Affiliated Hospital of Wenzhou Medical University, 1 Nan Bai Xiang Street, Wenzhou, Zhejiang 325035, P.R. China

E-mail: zjwzcj24@163.com

Key words: microRNA, miR-187, gastric cancer, mitotic arrest deficient-like 2, stomatin (EPB72)-like 2 development and progression of GC. Moreover, miR-187 may be a potential biomarker and therapeutic target in GC.

\section{Introduction}

Gastric cancer (GC) is one of the most common malignancies, and is considered as the second leading cause of cancer-related mortality worldwide (1). Although the five-year post-operative survival rate of GC has improved to some extent and concordantly with advances made in specific treatment, the prognosis for GC remains very poor. Further clarification of the mechanism underlying GC development from the molecular level and seeking of novel biological cancer markers and effective therapeutic targets may aid in improving the prognosis of GC.

microRNAs (miRNAs) are a class of endogenous, small non-coding regulatory RNAs that are 19-25 nucleotides in length, and are known to be involved in the regulation of gene expression by repressing translation or by decreasing the stability of mRNAs (2). In total, $>2,578$ types of miRNA have been discovered, and they regulate $\sim 1 / 3$ of human protein-encoding genes (3). Accumulating evidence has indicated that miRNAs are important in numerous crucial biological processes, including cell proliferation, apoptosis and energy metabolism $(2,4,5)$. Moreover, it has been previously reported that $\sim 50 \%$ of miRNAs are located in the chromosomal regions that are known to be frequently deleted or amplified in human cancer cells (6). Previous studies revealed that miRNAs may function as oncogenes or tumor suppressors that are involved in tumorigenesis $(7,8)$. In addition, emerging evidence has confirmed that aberrant expression of miRNAs in GC, including miR-21, miR-27a and miR-218, are involved in tumor growth, invasion and metastasis (9-11). These data have indicated the importance of miRNAs in GC development and have provided insights into the mechanisms that underlie tumorigenesis.

miR-187 has been revealed to be associated with numerous neoplasms $(12,13)$. However, mir-187 is differentially expressed between normal and different cancer tissues, which has revealed the various effects of prognostic evaluation (13). Mulrane et al (12) reported that miR-187 expression in breast cancer leads to a more aggressive, rapidly progressing and invasive phenotype, which serves as an independent predictor of the outcome. However, Zhao et al (13) revealed that miR-187 
was downregulated in renal cell carcinoma tissues, and lower miR-187 expression levels were associated with higher tumor grade and staging. To date, the expression of miR-187 and its clinical significance in GC remain poorly understood.

In the present study, the expression and clinical significance of miR-187 in the tissues of GC were explored. Cell proliferation in vitro and cell cycle phase analysis were further investigated by the ectopic expression of miR-187 in a GC cell-line. Furthermore, the potential target genes and their expression levels were determined. The observations of the present study may assist in the elucidation of the functions and pathophysiological roles of miRNA-187 and provide a formal basis for further research into the role of mir-187 in gastric carcinoma.

\section{Materials and methods}

Patients and tumor tissues. In total, 32 pairs of human gastric tissue samples were obtained from patients who underwent surgical resection in the Department of Gastrointestinal Surgery at the First Hospital of Wenzhou Medical College (Wenzhou, China) between 2008 and 2012. Patients were aged between 42 and 80 years, with a mean age of 65 years, and were diagnosed with GC based on histopathological evaluation. The matched normal adjacent tissue was obtained from a segment of the resected specimens that was the farthest from the tumor (i.e., $>5 \mathrm{~cm}$ ). The samples were snap-frozen in liquid nitrogen and stored at $-80^{\circ} \mathrm{C}$. None of the patients received chemotherapy or radiotherapy prior to surgical excision. In addition, the tumor histological grade was staged using the TNM staging of the International Union against Cancer/American Joint Committee on Cancer (AJCC) system (2002) (14). The study was then approved by the Research Ethics Committee of Wenzhou Medical College, and written informed consent was obtained from all patients.

Reverse transcription-quantitative polymerase chain reaction (RT-qPCR) quantification of $m i R-187$. Total RNA was extracted from the specimens using TRIzol reagent (cat. no. 15596026; Invitrogen; Thermo Fisher Scientific, Inc., Waltham, MA, USA) following the manufacturer's instructions. In order to remove potentially contaminated DNA, the extracted RNA samples were further purified using DNase I reagent (Thermo Fisher Scientific, Inc.) according to the manufacturer's protocol. A NanoDrop2000 spectrophotometer (Thermo Fisher Scientific, Inc.) detected RNA purity by measuring the $\mathrm{OD}_{260} / \mathrm{OD}_{280}$ ratio and calculating the RNA concentration and purity. Only an $\mathrm{OD}_{260} / \mathrm{OD}_{280}$ ratio $>1.8$ was considered suitable for subsequent experiments. At last, the integrity of the RNA was checked by $1.0 \%$ denaturing agarose gel electrophoresis (Bio-Rad Laboratories, Inc., Hercules, CA, USA). Xue et al (15) reported a method of detecting miRNA by stem-loop qPCR, where GAPDH RNA was used as an endogenous control. Using this approach, the expression of miR-187 was analyzed in tissue samples. The first-strand cDNA synthesis was performed with the ReverTra Ace ${ }^{\circledR}$ qPCR RT kit (cat. no. FSQ-101; Toyobo Co., Ltd., Osaka, Japan) using $0.5 \mu \mathrm{g}$ total RNA as the template and specific reverse primers at $16^{\circ} \mathrm{C}$ for $30 \mathrm{~min}, 42^{\circ} \mathrm{C}$ for $30 \mathrm{~min}$ and $95^{\circ} \mathrm{C}$ for $5 \mathrm{~min}$ of reverse transcription. The resulting cDNA was amplified by PCR using miRNA specific primers with
SYBR $^{\circledR}$ Green Real-Time PCR Master Mix (Toyobo Co., Ltd.) and was performed using an Eppendorf Mastercycler Ep Realplex (SABiosciences; Qiagen Sciences, Inc., Gaithersburg, MD, USA). The primers used for qPCR are listed in Table I. PCR parameters were as follows: $95^{\circ} \mathrm{C}$ for $5 \mathrm{~min}$, followed by 40 cycles of $95^{\circ} \mathrm{C}$ for $10 \mathrm{sec}, 60^{\circ} \mathrm{C}$ for $20 \mathrm{sec}$ and $72^{\circ} \mathrm{C}$ for $20 \mathrm{sec}$. At the end of the PCR cycles, a melting curve analysis was performed, and PCR products were analyzed using $2.5 \%$ PAGE electrophoresis.

The relative expression level of miR-187 in tissues was compared to the endogenous control or paired normal tissues. Thus, the $2^{-\Delta \mathrm{Cq}}$ and $2^{-\Delta \Delta \mathrm{Cq}}$ methods were used to evaluate the relative expression levels of the target (16). In these analyses; $\Delta \mathrm{Cq}=\mathrm{Cq}_{\mathrm{miR}-187}-\mathrm{Cq}_{\mathrm{GAPDH}}$, and $\Delta \Delta \mathrm{Cq}=\left[\left(\mathrm{Cq}_{\mathrm{miR}-187}-\mathrm{Cq}_{\mathrm{GAPDH}}\right)\right.$ tumor $]-\left[\left(\mathrm{Cq}_{\mathrm{miR}-187}-\mathrm{Cq}_{\mathrm{GAPDH}}\right)_{\text {control }}\right]$. When the data were calculated by the $\Delta \Delta \mathrm{Cq}$ method, the value of the relative expression ratio $<1.0$ was considered to be low expression in cancer relative to the control, and a ratio $>1.0$ was considered to indicate a high level of expression. Moreover, all qPCR assays were run in triplicate.

Cell line and culture conditions. MGC-803 is a poorly differentiated GC cell-line and was purchased from the Institute of Biochemistry and Cell Biology at the Chinese Academy of Sciences (Shanghai, China). MGC-803 cells were cultured in RPMI-1640 medium (Hyclone; GE Healthcare Life Sciences, Chalfont, UK) and were all supplemented with $10 \%$ fetal bovine serum (Hyclone; GE Healthcare Life Sciences), $50 \mathrm{U} / \mathrm{ml}$ penicillin-G (Invitrogen; Thermo Fisher Scientific, Inc.) and $50 \mu \mathrm{g} / \mathrm{ml}$ streptomycin sulfate (Invitrogen; Thermo Fisher Scientific, Inc.) at $37^{\circ} \mathrm{C}$ and $5 \% \mathrm{CO}_{2}$ in the air.

RNA oligoribonucleotides and cell transfection. The miRNA mimics were designed and synthesized by the GenePharma Company (Shanghai, China). miR-187 mimics (Table II) were an RNA duplex and negative control (NC) RNA duplex and were non-homologous to any human genomic sequences. All the oligonucleotide sequences used in this experiment are listed in Table II. The mimic transfection was performed with Lipofectamine 2000 reagent (Invitrogen; Thermo Fisher Scientific, Inc.) following the manufacturer's instructions. The GC cells were seeded into 6-well plates and were grown to $60-70 \%$ confluence before transfection. Moreover, transfection efficiency was then monitored using qPCR.

Cell Counting Kit-8(CCK-8) proliferation assay. The effect of miR-187 on the proliferation of GC cells was evaluated using the CCK-8 assay kit (Dojindo Molecular technologies, Inc., Kumamoto, Japan), according to the manufacturers instructions. MGC-803 cells were plated into 96 -well plates at a density of $8 \times 10^{3}$ cells/well in three parts. After $24 \mathrm{~h}$ of static culture, the cells were transfected with miR-187 mimics and the negative control (NC) using Lipofectamine 2000. The cellular proliferation capacity for the untransfected blank control (Blank), cells transfected with miR-187 mimic NCs and cells transfected with miRNA-187 mimics were measured using the CCK-8 assay. Moreover, the transfected miRNA-187 mimics group was set to final concentrations of 20,40 and $80 \mathrm{nM}$. The cells were then incubated for 24,48 and $72 \mathrm{~h}$ at $37^{\circ} \mathrm{C}$ in an atmosphere of $5 \% \mathrm{CO}_{2}$ in the air. Next, a total $10 \mu \mathrm{l}$ of the 
Table I. RT-qPCR primer sequences for the amplification of miR-187 and GAPDH.

\begin{tabular}{llll}
\hline Gene & \multicolumn{1}{c}{ Primer } & Sequence & Product (bp) \\
\hline miR-187 & RT stem-loop & 5'-GTCGTATCCAGTGCAGGGTCCGAGGT & 61 \\
& & ATTCGCACTGGATACGACCCGGCTGC-3' & \\
& Forward & 5'-TCGTGGGTCGTGTCTTGTGTTGC-3' \\
GAPDH & Reverse & 5'-GCAGGGTCCGAGGTATTC-3' & 101 \\
& Forward & 5'-CAGGGCTGCTTTTAACTCTGGTAA-3' \\
\hline
\end{tabular}

RT-qPCR, reverse transcription-quantitative polymerase chain reaction; miR, microRNA; GAPDH, glyceraldehyde 3-phosphate dehydrogenase; bp, base pair.

Table II. Sequence of miR-187 mimics and negative control.

\begin{tabular}{ll}
\hline RNA oligo & Sequence \\
\hline miR-187 mimic & 5'-UCGUGUCUUGUGUUGCAGCCGG-3' \\
& 3'-UUAGCACAGAACACAACGUCGG-5' \\
Negative control & 5'-UUCUCCGAACGUGUCACGUTT-3' \\
& 3'-TTAAGAGGCUUGCACAGUGCA-5' \\
\hline
\end{tabular}

CCK-8 solution was added to each well, and the cells were incubated for a further $4 \mathrm{~h}$. The absorbance (A) values were determined using a spectrophotometer (EPOCH2; BioTeK Instruments, Inc., Winooski, VT, USA) at a wavelength of $450 \mathrm{~nm}$, and the experiment was repeated three times. The inhibition rate of cell proliferation $=1-\left(\mathrm{A}_{450 \mathrm{~nm}}\right.$ of the study group $/ \mathrm{A}_{450 \mathrm{~nm}}$ of the control group) $\mathrm{x} 100 \%$. In addition, the relative activity of the cells $=A_{450 \mathrm{~nm}}$ of the study group $/ \mathrm{A}_{450 \mathrm{~nm}}$ of the control group.

Cell cycle analysis. For the cell cycle analysis, cells were seeded into 6 -well plates at a density of $2 \times 10^{5}$ cells/well, and transfected according to the protocol described above. The experimental groups and time points were set as described for the proliferation assay above. The cells were obtained after being cultured for an appropriate time by trypsinization, and were then pooled with the floating cells, centrifuged at $1,000 \mathrm{x} \mathrm{g}$ for $5 \mathrm{~min}$ at $4^{\circ} \mathrm{C}$ and stored at $-20^{\circ} \mathrm{C}$ overnight. DNA staining solution that contained (PI) iodide and RNaseA reagent [MultiSciences (Lianke) Biotech Co., Ltd., Hangzhou, China] were added to the cells. Moreover, cell cycle analysis was performed by fluorescence-activated cell sorting flow cytometry using CellQuest software 1.0 (BD Biosciences, San Jose, CA, USA).

Western blot analysis. miR-187 mimics (5'-UCGUGUCUU GUGUUGCAGCCGG-3'; Applied Biological Materials, Inc., Richmond, BC, Canada) or negative control oligonucleotides (5'-UUCUCCGAACGUGUCACGUTT-3'; Applied Biological Materials, Inc.) at a concentration of $40 \mathrm{nM}$ were transfected into GC MGC-803 cells using the Lipofectamine ${ }^{\mathrm{TM}} 2000$ Transfection Reagent kit (cat. no. 11668019; Invitrogen; Thermo Fisher Scientific, Inc.) and cells were harvested $48 \mathrm{~h}$ after transfection. Proteins were extracted and separated on a 12\% SDS-PAGE gel and transferred onto nitrocellulose membranes (Bio-Rad Laboratories, Inc.). The membrane was then blocked with 5\% non-fat milk proteins and incubated with anti-MAD2L2 (cat. no. ab180579; 1:2,000), anti-STOML2 (cat. no. ab37531; 1:2,000; each Abcam, Cambridge, UK) or anti- $\beta$-actin antibody (Sigma-Aldrich; Merck KGaA, Darmstadt, Germany). After being washed extensively, a goat anti-mouse secondary antibody (Pierce Biotechnology, Inc., Rockford, IL, USA) was added to the system. The proteins were detected using enhanced chemiluminescence reagents (cat. no. 32106; Pierce Biotechnology, Inc.). Protein expression levels were quantified using Image-Pro Plus 6.0 software (Media Cybernetics, Inc., Rockville, MD, USA).

Luciferase reporter assays. In order to verify the direct interaction of miR-187 to the target genes MAD2L2 and STOML2, human mRNA sequence were cloned into the pMIR-reporter construct (Ambion; Thermo Fisher Scientific, Inc.) in order to synthesize the luciferase reporter construct. Wild-type and mutant MAD2L2 and STOML2 mRNA fragments were amplified and sub-cloned into HindIII sites of the luciferase reporter, and luciferase reporter assays were performed as previously described (17). MGC803 cells were co-transfected with $50 \mathrm{nM}$ single-stranded miRNA mimics or internal control oligonucleotides, then plated into 24-well plates with $10 \mathrm{ng}$ pRL-TK (Promega Corporation, Madison, WI, USA) and $50 \mathrm{ng}$ firefly luciferase reporter using the JetPRIME reagent (Polyplus-transfection) according to the manufacturer's instructions. Cells were collected at $48 \mathrm{~h}$ after transfection (performed as aforementioned) and analyzed using the Dual-Luciferase Reporter Assay System (Promega Corporation).

Immunohistochemistry. Paired paraffin-embedded tissue sections $(n=20)$ were cut into $5-\mu \mathrm{m}$ sections, deparaffinized in xylene and rehydrated in graded series of ethanols followed by heat-induced epitope retrieval in citrate buffer ( $\mathrm{pH} 6.0$ ). The expression levels of MAD2L2 and STOML2 were detected using polyclonal antibodies targeted to MAD2L2 (cat. no. ab180579; 1:50) and STOML2 (cat. no. ab37531; 1:50; each Abcam). Next, samples were incubated with horseradish peroxidase-conjugated secondary goat anti-mouse antibodies (cat. no. SPKIT-C7; Fuzhou Maixin Biotech Co., 
Ltd., Fuzhou, China) and visualized with 3,3'-diaminodbenzidine (Beijing Zhongshan Golden Bridge Biotechnology Co., Ltd., Beijing, China). Images obtained were processed using Image-Pro Plus 6.0 software (Media Cybernetics, Inc., Rockville, MD, USA).

Statistical analysis. The expression of miR-187 was calculated for paired groups (i.e., the tumor and paired non-tumor) were compared using non-parametric Wilcoxon tests. The statistical significance of correlation between the expression of miR-187 and the clinicopathological parameters was calculated using non-parametric tests (Mann-Whitney U test between two groups and Kruskall-Wallis test for three or more groups). The association between miR-187 expression and prognosis was analyzed using the Kaplan-Meier survival curve statistics. In the Kaplan-Meier survival curve, high expression was defined as the fold-change of $>1$ and low expression levels as $<1$ compared to the probability of survival by the Log-rank test. Statistical analysis was performed using SPSS 17.0 software (SPSS, Inc., Chicago, IL, USA). Differences were considered statistically significant at a value of $\mathrm{P}<0.05$.

\section{Results}

Expression of miR-187 in GC tissues and matched non-tumor tissues. PCR products of miR-187 and GAPDH cDNA revealed a single band at the appropriate position (67 bp for miR-187 and 101 bp for GAPDH) on the electrophoretic gel (Fig. 1A). In addition, the melting curves of the products were sharply defined curves with a narrow peak (Fig. 1B). The combination of melting curves and gel electrophoresis confirmed PCR specificity. Furthermore, the expression of miR-187 in all 32 pairs of GC tissues and their matched non-cancerous tissues was detected using qPCR. As shown in Fig. 1C, the expression levels of miR-187 were lower in gastric tumors than in normal control tissues. The median relative expression levels of miR-187 was 0.031 (25-75th percentile, 0.013-0.102) in tumor samples, and was compared to that in non-tumor control samples, which was set at 0.086 (25-75th percentile, 0.028-0.192). Moreover, the difference in expression of miR-21 between the tumor and the control samples was statistically significant $(\mathrm{P}=0.037$, Wilcoxon test).

Association between the expression of $m i R-187$ and clinicopathologicalfeatures of $G C$. The association between miR-187 expression and the clinical and pathological characteristics of GC were explored further (Table III). The downregulated expression levels of miR-187 were associated with cell differentiation $(\mathrm{P}=0.042)$ and TNM stage $(\mathrm{P}=0.034)$ in $\mathrm{GC}$ patients. However, no significant association was identified between the expression of miR-187 and the demographic or clinical variables, including gender, age, tumor location, tumor size, depth of tumor invasion and lymph node metastasis $(\mathrm{P}>0.05)$. According to the relative expression levels of miR-187 of paired tumor and normal tissues, specimens with a relative expression level $<1$ were set as Group One, and specimens with a relative value $\geq 1$ were set as Group Two. A Kaplan-Meier survival analysis illustrated that the cohort with higher expression levels of miR-187 demonstrated greater rates of survival than those with lower levels of expression of miR-187 (Fig. 2).
Moreover, the 5-year survival rate for Group One was $66.7 \%$, while that for Group Two was $13.5 \%$.

miR-187 inhibited cell proliferation and induced cell cycle arrest at the $G_{0} / G_{1}$ phase in vitro. The significant reduction in miR-187 expression in GC samples prompted us to explore the possible biological roles of miR-187 in the mechanism of tumorigenesis. Thus, the effect of miR-187 expressing again was investigated on the proliferative capacity of the GC cell-line. Compared with that of the cells transfected with either the negative or blank control, MGC-803 cells that were transiently transfected with miR-187 mimics had a significant growth inhibition to different degrees $(\mathrm{P}<0.05$; Fig. 3A). Moreover, the cell vitality of GC cells was markedly associated with different transfected concentrations and was time-dependent $(\mathrm{P}<0.05$; Fig. 3A). In order to investigate further whether inhibition of MGC-803 proliferation reflected cell cycle arrest, the progression of cell cycle phases was analyzed by PI staining and flow cytometry. The results revealed that MGC-803 cells that were dose-dependently transfected with $\mathrm{miR}-187$ mimics had an evident effect on cell cycle arrest at the $G_{0} / G_{1}$ phase (Fig. 3B and C) as compared with the blank and the negative control group $(\mathrm{P}<0.05)$. However, the proportion of cells that were transfected with different doses of miR-187 mimics had little effect on the cell cycle phases $(\mathrm{P}>0.05)$.

miR-187 targets MAD2L2 and STOML2. As miRNAs function mainly through the inhibition of target genes, the target of miR-187 that functions in the pathogenesis of GC was further analyzed. Based on previously published CLASH data, which provided direct experimental data of miRNA-targeted pairs (18), MAD2L2, STOML2 and tubulin, $\gamma 1$ (TUBG1) were identified as potential targets of miR-187. In order to confirm whether these genes that had been regulated by miR-187 were involved in the pathogenesis of GC, MGC-803 cells were transfected with miR-187 mimics or negative control oligonucleotides, and the protein expression levels of the genes were examined by western blotting. Data indicated that the expression of MAD2L2 and STOML2 were consistently and substantially downregulated by the ectopic expression of miR-187, whereas TUBG1 expression was not significantly affected by miR-187 (Fig. 4A).

Luciferase reporter assay. In order to verify the interaction between miR-187, MAD2L2 and STOML2, luciferase reporter assays were performed in MGC803 cells. The luciferase reporter plasmid with sequences of MAD2L2 and STOML2 mRNA or mutant sequences were co-transfected into MGC803 cells for $48 \mathrm{~h}$ with miR-187 or NC, respectively. Moreover, the luciferase activity was measured in transfected cells. The results demonstrated that the reporter plasmid of MAD2L2 and STOML2 mRNA caused a significant decrease in the luciferase activity in cells that were transfected with miR-187. By contrast, the luciferase activity of the reporter plasmid with mutant sequences of MAD2L2 and STOML2 did not change (Fig. 4B). Furthermore, GC tissues with a low miR-187 demonstrated much higher MAD2L2 and STOML2 expression when compared with paired normal gastric tissues (Fig. 5). Altogether, the results suggested that MAD2L2 and STOML2 was the target of miR-187 in GC cells. 


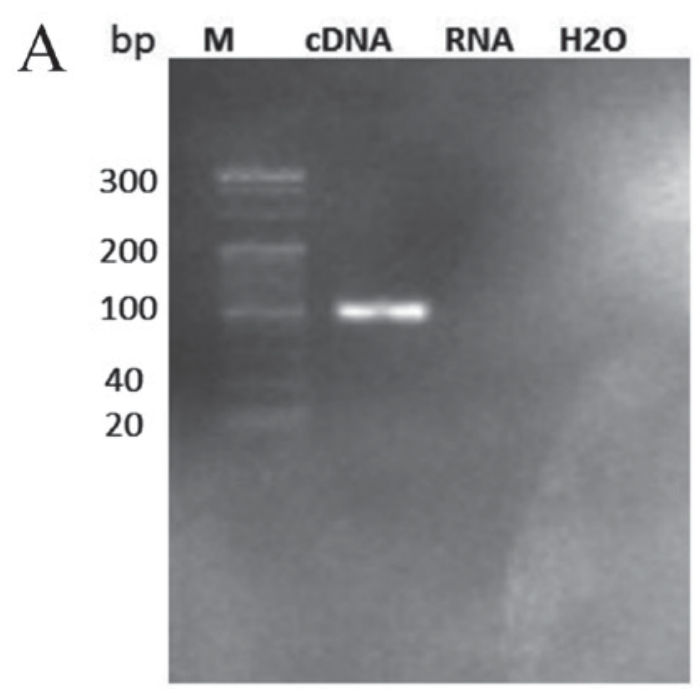

GAPDH

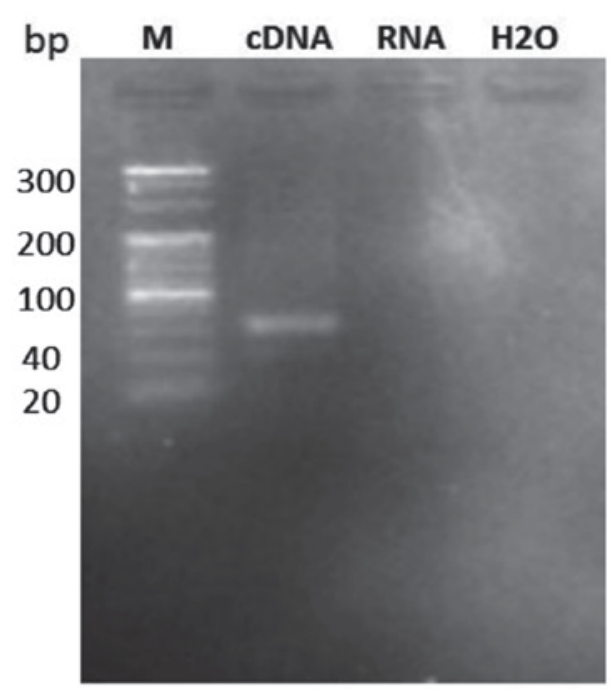

miR-187

\section{B}

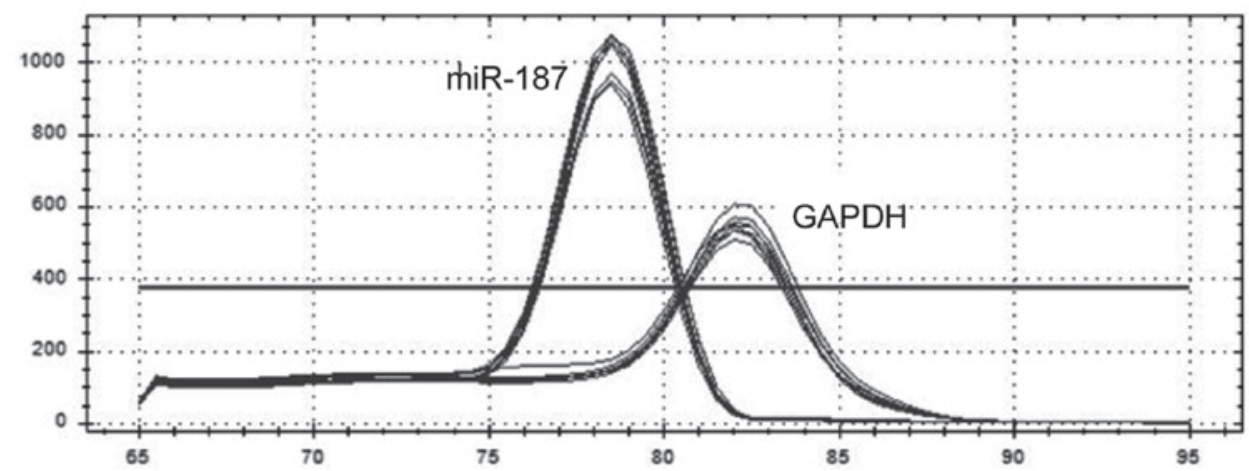

$\mathrm{C}$

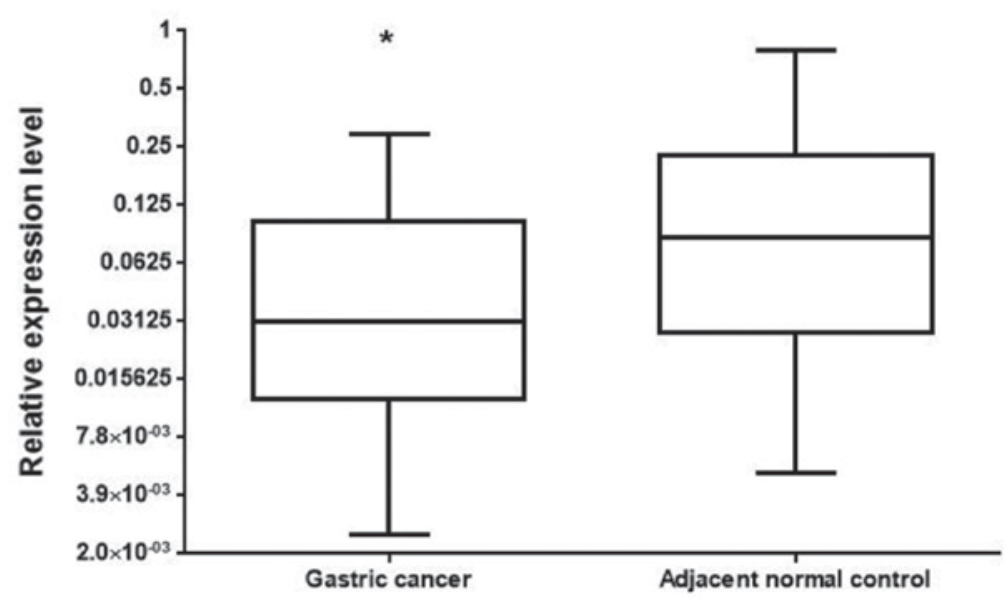

Figure 1. miR-187 was downregulated in 32 pairs of human gastric tissue samples. (A) PCR products of miR-187 and endogenous control GAPDH (Lane M, 20 bp DNA marker; lane RNA, RNA control; lane $\mathrm{H}_{2} \mathrm{O}$, Negative control). (B) Melting curve of miR-187 RT-qPCR amplified products. (C) Expression of miR-187 was measured by RT-qPCR, and the expression levels of miR-187 were normalized against GAPDH RNA expression for subsequent analyses. A P-value was obtained using a one-tailed Student's t-test. ${ }^{*} \mathrm{P}<0.05$ vs. the control. RT-qPCR, reverse transcription-quantitative polymerase chain reaction; GAPDH, glyceraldehyde 3-phosphate dehydrogenase.

\section{Discussion}

Accumulating evidence has indicated that the abnormal expression of miR-187 is closely associated with cancer cell proliferation and apoptosis, and that they may serve as tumor suppressors or oncogenes $(19,20)$. Previously, numerous studies have shown that miR-187 is anomalously expressed in various tumor types, including thyroid (21), nasopharyngeal carcinoma (22), esophageal (23) and pancreatic cancer (24) and neuroblastoma cell tumors (25). However, in different cancers, 
Table III. Association between the expression of miR-187 with clinicopathological features of gastric cancer.

\begin{tabular}{|c|c|c|c|}
\hline Clinical characteristics & Cases (n) & $\begin{array}{l}\text { miR-187 } \\
\text { expression }^{\text {a }}\end{array}$ & P-value \\
\hline Age (years) & & & 0.258 \\
\hline$<60$ & 8 & $0.60 \pm 0.63$ & \\
\hline$\geq 60$ & 24 & $0.97 \pm 0.89$ & \\
\hline Sex & & & 0.630 \\
\hline Male & 23 & $0.86 \pm 0.87$ & \\
\hline Female & 9 & $0.91 \pm 0.82$ & \\
\hline Tumor size $(\mathrm{cm})$ & & & 0.440 \\
\hline$<5$ & 26 & $0.88 \pm 0.91$ & \\
\hline$\geq 5$ & 6 & $0.87 \pm 0.53$ & \\
\hline Location & & & 0.967 \\
\hline Upper area & 4 & $0.59 \pm 0.17$ & \\
\hline Middle area & 7 & $0.77 \pm 0.87$ & \\
\hline Lower area & 21 & $0.96 \pm 0.92$ & \\
\hline T stage & & & 0.500 \\
\hline $\mathrm{T} 1 / \mathrm{T} 2$ & 7 & $1.21 \pm 1.00$ & \\
\hline $\mathrm{T} 3$ & 10 & $0.72 \pm 0.63$ & \\
\hline $\mathrm{T} 4$ & 15 & $0.82 \pm 0.89$ & \\
\hline Cell differentiation & & & 0.042 \\
\hline $\begin{array}{l}\text { Well or Moderately- } \\
\text { differentiated }\end{array}$ & 9 & $1.45 \pm 0.92$ & \\
\hline Poorly-differentiated & 23 & $0.65 \pm 0.71$ & \\
\hline Lymph node metastasis & & & 0.090 \\
\hline No & 9 & $1.21 \pm 0.86$ & \\
\hline Yes & 23 & $0.74 \pm 0.82$ & \\
\hline $\mathrm{N}$ stage & & & 0.270 \\
\hline N1 & 3 & $1.65 \pm 1.18$ & \\
\hline $\mathrm{N} 2$ & 12 & $0.65 \pm 0.85$ & \\
\hline N3 & 8 & $0.52 \pm 0.34$ & \\
\hline TNM stage & & & 0.034 \\
\hline $\mathrm{I} / \mathrm{II}$ & 11 & $1.23 \pm 0.84$ & \\
\hline III/IV & 21 & $0.69 \pm 0.80$ & \\
\hline
\end{tabular}

${ }^{a}$ Mean of relative expression \pm standard deviation. miR, microRNA; TNM, tumor node metastasis.

the changes in expression of miR-187 varied greatly, indicating that it may be involved in carcinogenesis and progression in a specific way.

A previous study demonstrated that miR-187 that was downregulated in tumor tissue was involved in ovarian cancer progression (26). Mulrane et al (12) revealed that the expression levels of miR-187 in breast cancer tissue was correlated with breast cancer invasion, and could be used as an independent prognostic factor. However, the expression level and the biological impact of miR-187 in GC remain unclear. In order to investigate the association of miR-187 and GC pathogenesis, qPCR was used to profile the expression of miR-187 in 32 matched gastric tumor tissues, and clarified the association between miR-187 and the clinicopathological characteristics of GC.

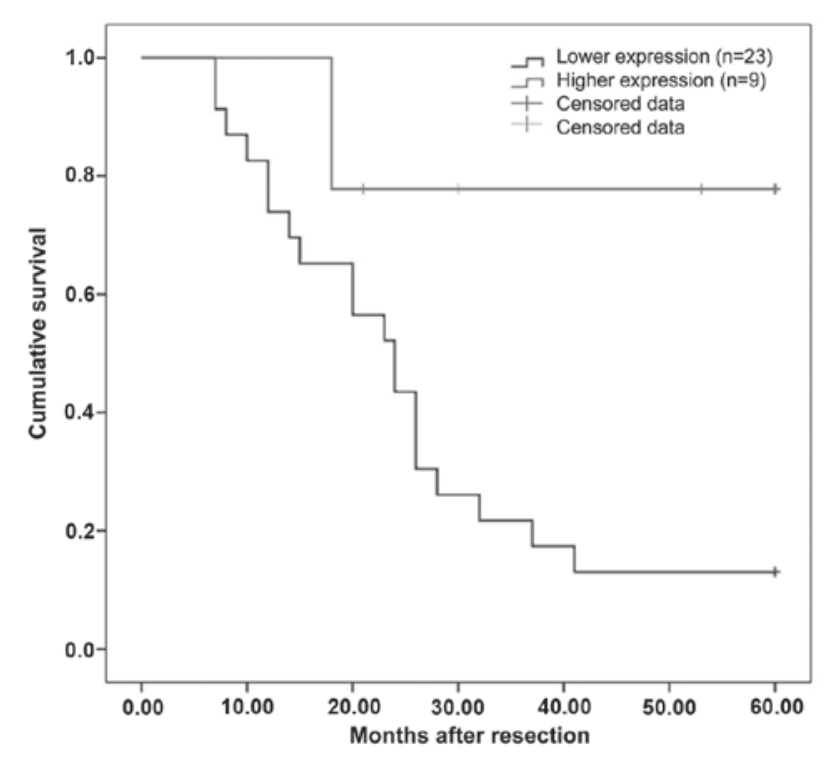

Figure 2. Analysis of the survival curves. Gastric cancer patients with high and low expression of miR-187 demonstrated differential survival statistics.

In the present study, the expression of miR-187 was found to be downregulated in gastric tumors compared to non-tumor tissues. Besides, miR-187 expression was associated with cell differentiation and TNM staging in GC patients. In addition, the Kaplan-Meier survival analysis illustrated that the cohort with a higher expression of miR-187 demonstrated higher survival rates than those cells with lower levels. These results indicated that miR-187 is important in the development and progression of GC. In view of the above, miR-187 is speculated to function as a tumor suppressor in GC, and the downregulation of miR-187 may promote its occurrence and development of GC.

To further clarify this point, the biological function of miR-187 was investigated. Deregulated cell proliferation is a key biological characteristic of neoplastic progression (27). In the present study, miR-187 mimics were transfected into MGC-803 cells, then the cell proliferation ability was evaluated. The CCK- 8 assay data indicated that the overexpression of miR-187 could suppress the proliferation of GC cells. However, the reason for miR-187 induced inhibition of cell proliferation remains to be investigated. Overall, cell cycle arrest was likely to be an important factor $(28,29)$. Indeed, the subsequent cell cycle analyses of the present study revealed that MGC-803 cells that were transfected with miR-187 mimics had an evident cell cycle arrest at the $G_{0} / G_{1}$ phase. Nevertheless, the proportion of cells of the different transfected groups demonstrated no difference. It is likely that the process of cell cycle arrest induced by miR-187 was influenced by another molecular mechanism since indirect regulation and miRNA may have complex roles that may formally involve multiple aspects and factors in cell cycle regulation.

The main function of miRNA is to regulate target gene expression by direct cleavage of the mRNA or by inhibition of protein synthesis according to the degree of complementarities with the 3 'untranslated region of target genes $(30,31)$. Perfect or nearly perfect base-pairing induced targeted mRNA cleavage whereas imperfect base pairing induced mainly translational 

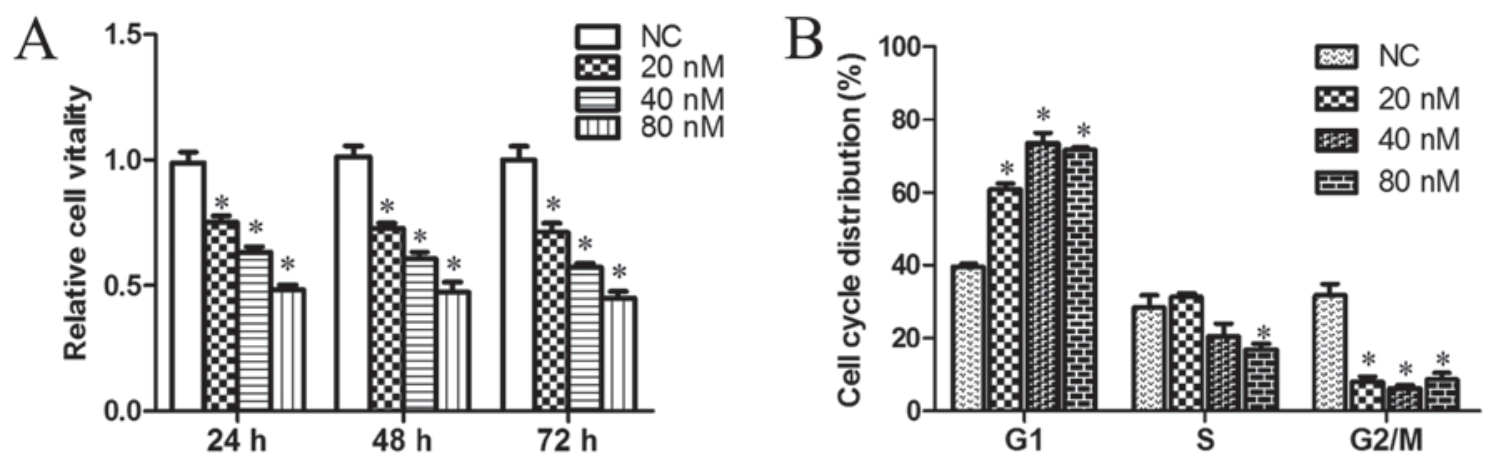

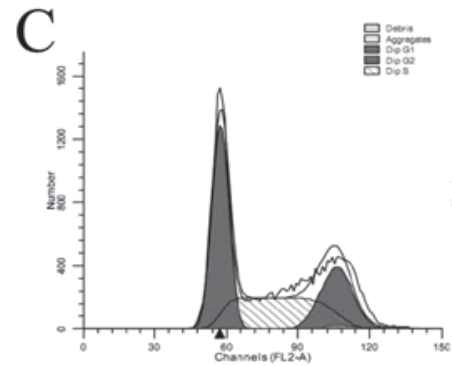

NC

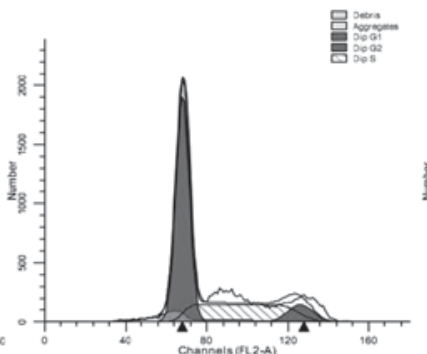

$20 \mathrm{nM}$

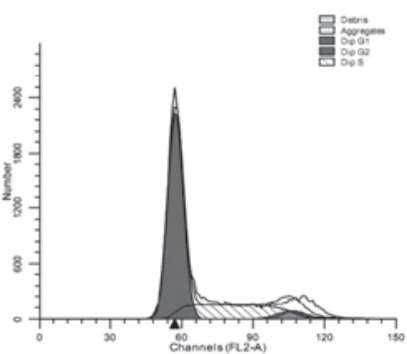

$40 \mathrm{nM}$

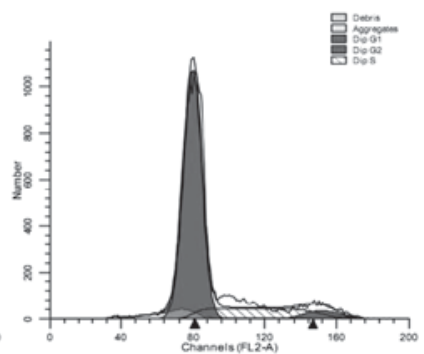

$80 \mathrm{nM}$

Figure 3. miR-187 restoration inhibited the viability of GC cells. (A) Relative cell vitality of the GC MGC-803 cell line was measured by the CCK-8 cell proliferation assay after being transfected with different concentrations of miR-187 mimics and at different time-points. (B and C) Cell-cycle distribution was determined by FACS analysis. MGC-803 cells were transfected with different concentrations of miR-187 mimics or negative control oligonucleotides for $24 \mathrm{~h}$. Data were subjected to Student's t-test. ${ }^{*} \mathrm{P}<0.05$ vs. NC group. NC, negative control; CCK-8, Cell Counting Kit-8; FACS, fluorescence-activated cell sorting.
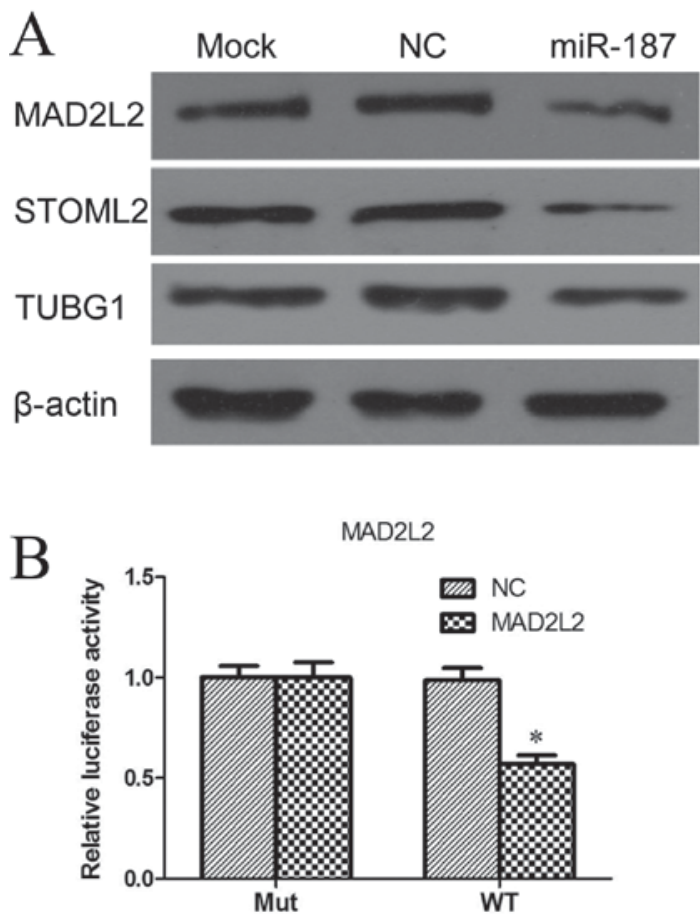

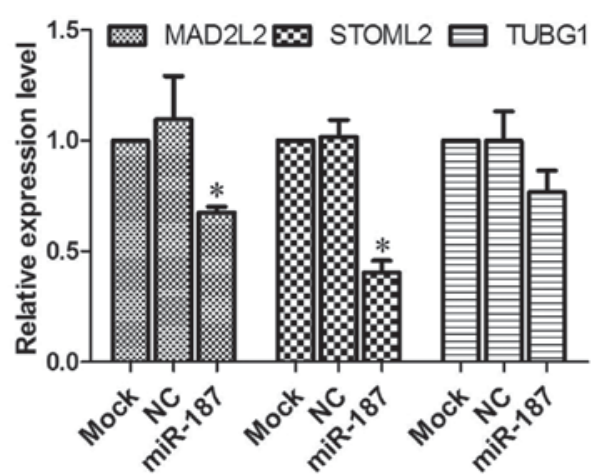

STOML2

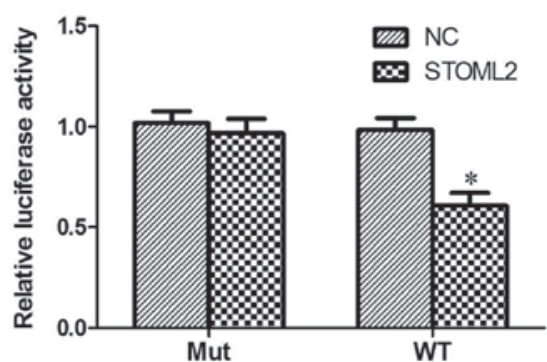

Figure 4. Effects of miR-187 on the protein expression levels of potential target genes. (A) In total, $48 \mathrm{~h}$ after transfection with the $40 \mathrm{nM}$ miR-187 mimics or NC oligonucleotides, MGC-803 cells were analyzed by western blotting. (B) Analysis of the luciferase activity. MGC 803 cells were co-transfected with miR-187 mimics and the firefly luciferase reporter plasmid containing putative miR-187 targeting sequences of MAD2L2 and STOML2. The plasmid construct pRL-TK served as an internal control. The normalized luciferase activity of the control oligonucleotides was set as the relative luciferase activity. " $\mathrm{P}<0.05$ vs. blank (mock) or NC groups. MAD2L2, mitotic arrest deficient-like 2; STOML2, stomatin (EPB72)-like 2; TUBG1, tubulin, gamma 1; NC, negative control; WT, wild type.

silencing of the target (32). Chao et al (26) demonstrated that miR-187 inhibited the epithelial-mesenchymal transition process and migration by targeting Dab2 in ovarian cancer cells. Furthermore, Mulrane et al (12) reported that miR-187 
A

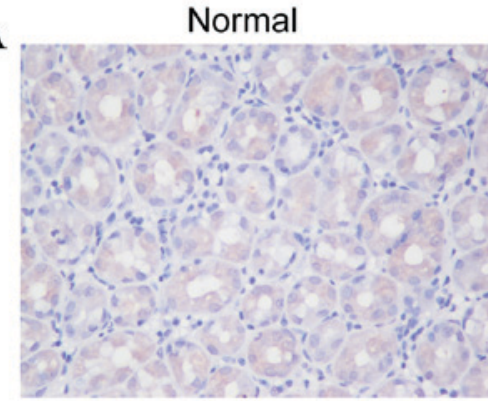

B

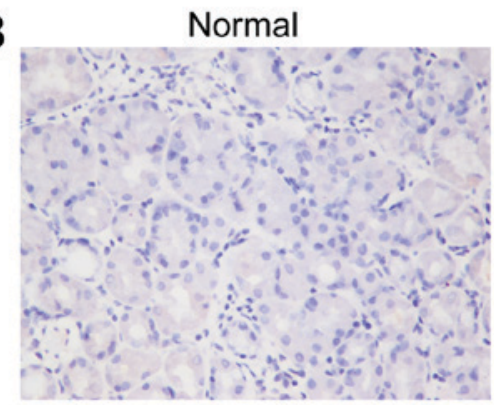

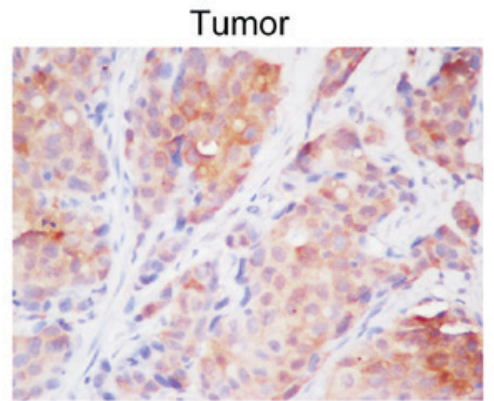

Tumor

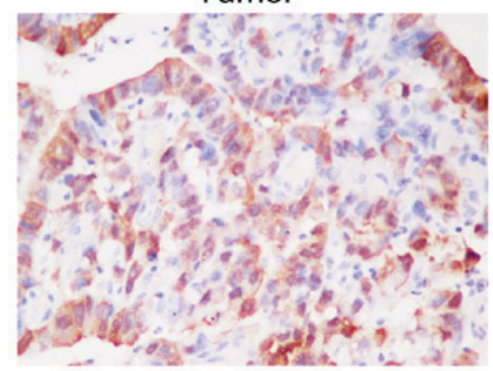

MAD2L2

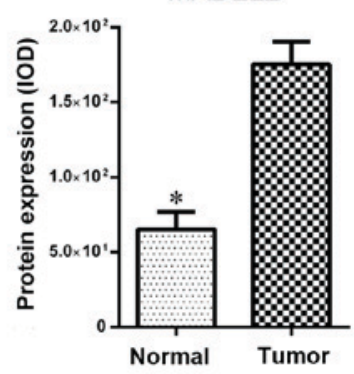

STOML2

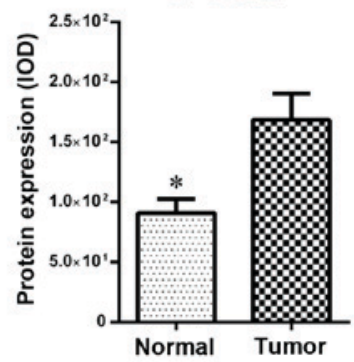

Figure 5. MAD2L2 and STOML2 were highly expressed in human gastric cancer tissues. Expression of (A) MAD2L2 and (B) STOML2 in gastric cancer tissues were determined by immunohistochemistry (magnification, $x 400$ ). Normal gastric tissue served as the control (magnification, $x 400)$. The protein expression levels were measured in different tissues by semi-quantitative immunohistochemistry $(\mathrm{n}=20)$. " $\mathrm{P}<0.05$ vs. tumor group. MAD2L2, mitotic arrest deficient-like 2; STOML2, stomatin (EPB72)-like 2; IOD, integrated optical density.

regulated collagenase (matrix metallopeptidase 13) expression by either direct or indirect pathways in breast cancer, and that this pathway may have also been involved in the migration of tumor cells. Moreover, Sirotkin et al (33) revealed that overexpression of miR-187 could reduce the expression of the apoptosis-related B-cell lymphoma 2 -associated X protein and the proliferation marker proliferating cell nuclear antigen in human ovarian granulosa cells. However, whether or not other target genes are responsible for the effect in GC is currently unknown.

A recent advance in high-throughput direct mapping of miRNA-mRNA binding sites from CLASH experiments directly identified the miRNA-mRNA target pairs that were associated with the human AGO1 protein, and presents an improved determination of the miRNA target (18). Based on the CLASH experiment data and combined with the analysis of miR-187 interference, MAD2L2 and STOML2 were consistently and substantially downregulated by the ectopic expression of miR-187.

In the present study, luciferase reporter assays further confirmed the interaction between miR-187, MAD2L2 and STOML2. Thus, MAD2L2 and STOML2 could be considered to be potential targets that were involved in the development of GC. It has been demonstrated that MAD2L2, which is a member of a family of genes involved in mitotic checkpoint control mechanisms, were significantly upregulated in colon cancer as compared to matched normal tissue (34). In addition, tumors that show upregulated MAD2L2 expression had significantly higher numbers of aberrant mitotic figures (anaphase bridges), an indication of chromosomal instability and a poor prognosis in colorectal cancer (34). STOML2 is a member of the stomatin superfamily, and has been identified as an oncogenic-related protein, whose functional expression is enhanced in numerous cancer types (35-38). Moreover, overexpression of STOML2 has been reported in the tissues of GC compared with the adjacent normal gastric epithelium. In addition, a high-level STOML2 expression was significantly correlated with the depth of invasion, lymph node and distant metastasis, and staging according to the AJCC and poor prognosis in GC (39).

The present data revealed that the expression of MAD2L2 and STOML2 were significantly inhibited in MGC-803 cells transfected with miR-187 mimics. Furthermore, high levels of expression of MAD2L2 and STOML2 were also confirmed in GC tissues compared with adjacent normal tissues. These results provided insights into the regulatory mechanism of miR-187 in GC. Downregulation of miR-187 in GC cells resulted in enhanced expression of its target genes, which may consequently favor tumor progression. However, miRNA displays multi-target characteristics, and has complex regulatory networks that are involved in numerous aspects of biological functions $(2,40,41)$. Therefore, the consequent molecular mechanism of miR-187 remains to be further investigated.

In conclusion, the present study demonstrated that there was a significantly reduced expression of miR-187 in GC tissues compared with their non-tumor counterparts. Moreover, the expression of miR-187 was associated with the degree of cell differentiation, pathological staging and prognosis in GC patients. The data of the present study also indicated that miR-187 could inhibit cell proliferation in vitro, and could induce cell cycle arrest at the $\mathrm{G}_{0} / \mathrm{G}_{1}$ phase. To the best of our knowledge, the present study confirmed the tumor suppressive roles of miR-187 in GC for the first time, and provided evidence for the potential utility of miR-187 as a biomarker and therapeutic target against GC. 


\section{Acknowledgements}

Not applicable.

\section{Funding}

The present study was supported by grants from the National Natural Science Foundation of China (grant nos. 81472308 and 31470891), the Zhejiang Provincial Natural Science Foundation of China (grant nos. Y2100909 and LY12H05003), the Jinhua Science and Technology Bureau (grant no. 2012-3-005) and the Zhejiang Science and Technology Bureau (grant nos. 20 12C33126, 2012C37080 and 2010C33094). These sponsors provided funding for the experiments and the collection of specimens.

\section{Availability of data and materials}

The datasets used and/or analyzed during the current study are available from the corresponding author on reasonable request.

\section{Authors' contributions}

All authors have approved this manuscript and agreed to be accountable for all aspects of this work. WC, JC and SS contributed in the conception and design and revision of the manuscript, the acquisition, analysis and interpretation of data. XS and LZ prepared the initial draft of the manuscript, contributed in the acquisition, analysis and interpretation of data, and helped perform the cell biology experiments. YC, JW and YY made substantial contributions to conception and design, acquisition of data, and analysis of data, and performed the western blot experiments.

\section{Ethics approval and consent to participate}

The present study was approved by the Research Ethics Committee of Wenzhou Medical College and written informed consent was obtained from all patients.

\section{Consent for publication}

Not applicable.

\section{Competing interests}

The authors declare that they have no competing interests.

\section{References}

1. Crew KD and Neugut AI: Epidemiology of gastric cancer. World J Gastroenterol 12: 354-362, 2006.

2. Bartel DP: MicroRNAs: Genomics, biogenesis, mechanism, and function. Cell 116: 281-297, 2004.

3. Chan JA, Krichevsky AM and Kosik KS: MicroRNA-21 is an antiapoptotic factor in human glioblastoma cells. Cancer Res 65: 6029-6033, 2005.

4. Care A, Catalucci D, Felicetti F, Bonci D, Addario A, Gallo P, Bang ML, Segnalini P, Gu Y, Dalton ND, et al: MicroRNA-133 controls cardiac hypertrophy. Nat Med 13: 613-618, 2007.
5. Wan HY, Guo LM, Liu T, Liu M, Li X and Tang H: Regulation of the transcription factor NF-kappaB1 by microRNA-9 in human gastric adenocarcinoma. Mol Cancer 9: 16, 2010.

6. Calin GA, Sevignani C, Dumitru CD, Hyslop T, Noch E, Yendamuri S, Shimizu M, Rattan S, Bullrich F, Negrini M and Croce CM: Human microRNA genes are frequently located at fragile sites and genomic regions involved in cancers. Proc Natl Acad Sci USA 101: 2999-3004, 2004.

7. Volinia S, Calin GA, Liu CG, Ambs S, Cimmino A, Petrocca F, Visone R, Iorio M, Roldo C, Ferracin M, et al: A microRNA expression signature of human solid tumors defines cancer gene targets. Proc Natl Acad Sci USA 103: 2257-2261, 2006.

8. Calin GA and Croce CM: MicroRNA-cancer connection: The beginning of a new tale. Cancer Res 66: 7390-7394, 2006.

9. Liu T, Tang H, Lang Y, Liu M and Li X: MicroRNA-27a functions as an oncogene in gastric adenocarcinoma by targeting prohibitin. Cancer Lett 273: 233-242, 2009.

10. Tie J, Pan Y, Zhao L, Wu K, Liu J, Sun S, Guo X, Wang B, Gang Y, Zhang Y, et al: MiR-218 inhibits invasion and metastasis of gastric cancer by targeting the Robo1 receptor. PLoS Genet 6: e1000879, 2010.

11. Zhang Z, Li Z, Gao C, Chen P, Chen J, Liu W, Xiao S and Lu H: miR-21 plays a pivotal role in gastric cancer pathogenesis and progression. Lab Invest 88: 1358-1366, 2008.

12. Mulrane L, Madden SF, Brennan DJ, Gremel G, McGee SF, McNally S, Martin F, Crown JP, Jirström K, Higgins DG, et al: miR-187 is an independent prognostic factor in breast cancer and confers increased invasive potential in vitro. Clin Cancer Res 18: 6702-6713, 2012

13. Zhao J, Lei T, Xu C, Li H, Ma W, Yang Y, Fan S and Liu Y: MicroRNA-187, down-regulated in clear cell renal cell carcinoma and associated with lower survival, inhibits cell growth and migration though targeting B7-H3. Biochem Biophys Res Commun 438: 439-444, 2013.

14. Greene FL, Page DL, Fleming ID, Fritz AG, Balch CM, Haller DG and Morrow M, (eds): AJCC Cancer staging manual: TNM classification of malignant tumors. 6th edition. New York, Springer-Verlag, 2002.

15. Xue X, Sun J, Zhang Q, Wang Z, Huang Y and Pan W: Identification and characterization of novel microRNAs from Schistosoma japonicum. PLoS One 3: e4034, 2008.

16. Livak KJ and Schmittgen TD: Analysis of relative gene expression data using real-time quantitative PCR and the 2(-Delta Delta C(T)) method. Methods 25: 402-408, 2001.

17. Liu L and Eisenman RN: Regulation of c-Myc protein abundance by a protein phosphatase $2 \mathrm{~A}-\mathrm{Glycogen}$ synthase kinase 33-Negative feedback pathway. Genes Cancer 3: 23-36, 2012.

18. Helwak A, Kudla G, Dudnakova T and Tollervey D: Mapping the human miRNA interactome by CLASH reveals frequent noncanonical binding. Cell 153: 654-665, 2013.

19. Cheng AM, Byrom MW, Shelton J and Ford LP: Antisense inhibition of human miRNAs and indications for an involvement of miRNA in cell growth and apoptosis. Nucleic Acids Res 33: 1290-1297, 2005

20. Park SY, Lee JH, Ha M, Nam JW and Kim VN: miR-29 miRNAs activate $\mathrm{p} 53$ by targeting $\mathrm{p} 85 \alpha$ and CDC42. Nat Struct Mol Biol 16: 23-29, 2008.

21. Nikiforova MN, Tseng GC, Steward D, Diorio D and Nikiforov YE: MicroRNA expression profiling of thyroid tumors: Biological significance and diagnostic utility. J Clin Endocrinol Metab 93: 1600-1608, 2008.

22. Chen HC, Chen GH, Chen YH, Liao WL, Liu CY, Chang KP, Chang YS and Chen SJ: MicroRNA deregulation and pathway alterations in nasopharyngeal carcinoma. Br J Cancer 100: 1002-1011, 2009.

23. Wijnhoven B, Hussey DJ, Watson DI, Tsykin A, Smith C and Michael MZ; South Australian Oesophageal Research Group: MicroRNA profiling of Barrett's oesophagus and oesophageal adenocarcinoma. Br J Surg 97: 853-861, 2010.

24. Bloomston M, Frankel WL, Petrocca F, Volinia S, Alder H, Hagan JP, Liu CG, Bhatt D, Taccioli C and Croce CM: MicroRNA expression patterns to differentiate pancreatic adenocarcinoma from normal pancreas and chronic pancreatitis. JAMA 297: 1901-1908, 2007.

25. Chen Y and Stallings RL: Differential patterns of microRNA expression in neuroblastoma are correlated with prognosis, differentiation, and apoptosis. Cancer Res 67: 976-983, 2007. 
26. Chao A, Lin CY, Lee YS, Tsai CL, Wei PC, Hsueh S, Wu TI, Tsai CN, Wang CJ, Chao AS, et al: Regulation of ovarian cancer progression by microRNA-187 through targeting Disabled homolog-2. Oncogene 31: 764-775, 2011.

27. Evan GI and Vousden KH: Proliferation, cell cycle and apoptosis in cancer. Nature 411: 342-348, 2001.

28. Sánchez CA, Rodríguez E, Varela E, Zapata E, Paez A, Massó FA, Montaño LF and Lóopez-Marure R: Statin-induced inhibition of MCF-7 breast cancer cell proliferation is related to cell cycle arrest and apoptotic and necrotic cell death mediated by an enhanced oxidative stress. Cancer Invest 26: 698-707, 2008.

29. Park WH, Lee YY, Kim ES, Seol JG, Jung CW, Lee CC and Kim BK: Lovastatin-induced inhibition of HL-60 cell proliferation via cell cycle arrest and apoptosis. Anticancer Res 19: 3133-3140, 1999

30. Zamore PD and Haley B: Ribo-gnome: The big world of small RNAs. Science 309: 1519-1524, 2005.

31. Mattick JS and Makunin IV: Non-coding RNA. Hum Mol Genet 15: R17-R29, 2006

32. He L and Hannon GJ: MicroRNAs: Small RNAs with a big role in gene regulation. Nat Rev Genet 5: 522-531, 2004.

33. Sirotkin AV, Lauková M, Ovcharenko D, Brenaut $P$ and Mlynček M: Identification of microRNAs controlling human ovarian cell proliferation and apoptosis. J Cell Physiol 223: 49-56, 2010.

34. Rimkus C, Friederichs J, Rosenberg R, Holzmann B, Siewert JR and Janssen KP: Expression of the mitotic checkpoint gene MAD2L2 has prognostic significance in colon cancer. Int J Cancer 120: 207-211, 2007.

35. Cao W, Zhang B, Liu Y, Li H, Zhang S, Fu L, Niu Y, Ning L, Cao X, Liu Z and Sun B: High-level SLP-2 expression and HER-2/neu protein expression are associated with decreased breast cancer patient survival. Am J Clin Pathol 128: 430-436, 2007.
36. Cao WF, Zhang LY, Liu MB, Tang PZ, Liu ZH and Sun BC: Prognostic significance of stomatin-like protein 2 overexpression in laryngeal squamous cell carcinoma: Clinical, histologic, and immunohistochemistry analyses with tissue microarray. Hum Pathol 38: 747-752, 2007.

37. Wang Y, Cao W, Yu Z and Liu Z: Downregulation of a mitochondria associated protein SLP-2 inhibits tumor cell motility, proliferation and enhances cell sensitivity to chemotherapeutic reagents. Cancer Biol Ther 8: 1651-1658, 2009.

38. Zhang L, Ding F, Cao W, Liu Z, Liu W, Yu Z, Wu Y, Li W, Li Y and Liu Z: Stomatin-like protein 2 is overexpressed in cancer and involved in regulating cell growth and cell adhesion in human esophageal squamous cell carcinoma. Clin Cancer Res 12: 1639-1646, 2006.

39. Liu D, Zhang L, Shen Z, Tan F, Hu Y, Yu J and Li G: Increased levels of SLP-2 correlate with poor prognosis in gastric cancer. Gastric Cancer 16: 498-504, 2013.

40. Brennecke J, Stark A, Russell RB and Cohen SM: Principles of microRNA-target recognition. PLoS Biol 3: e85, 2005.

41. Brodersen $\mathrm{P}$ and Voinnet $\mathrm{O}$ : Revisiting the principles of microRNA target recognition and mode of action. Nat Rev Mol Cell Biol 10: 141-148, 2009.

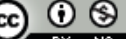

This work is licensed under a Creative Commons Attribution 4.0 International (CC BY-NC 4.0) License 